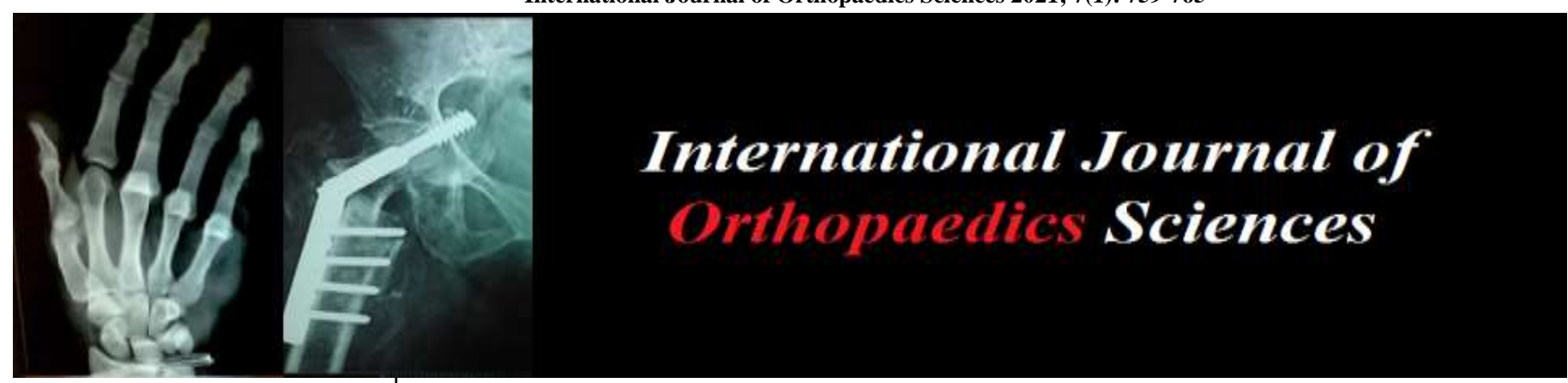

E-ISSN: 2395-1958

P-ISSN: 2706-6630

IJOS 2021; 7(1): 759-765

(C) 2021 IJOS

www.orthopaper.com

Received: 27-10-2020

Accepted: 14-12-2020

Dr. Daivik Shetty

Junior Resident, Department of

Orthopedics, AJ Institute of

Medical Sciences and Research

Centre, Karnataka, India

Dr. Mithun Shetty

Assistant Professor, Department of Orthopedics, AJ Institute of

Medical Sciences and Research

Centre, Karnataka, India

\section{Dr. Chinmaya SP}

Junior Resident, Department of Orthopedics, AJ Institute of

Medical Sciences and Research

Centre, Karnataka, India

Dr. Rithvik Rai

Intern, Department of

Orthopaedics, AJ Institute of

Medical Sciences and Research

Centre, Karnataka, India
Corresponding Author: Dr. Daivik Shetty Junior Resident, Department of Orthopedics, AJ Institute of Medical Sciences and Research Centre, Karnataka, India

\section{A case that gives you a cold shoulder - bilateral posterior fracture dislocation of shoulder after electrical shock: A rare case report}

\author{
Dr. Daivik Shetty, Dr. Mithun Shetty, Dr. Chinmaya SP and Dr. Rithvik \\ Rai
}

DOI: $\underline{\text { https://doi.org/10.22271/ortho.2021.v7.i11.2567 }}$

\begin{abstract}
Introduction: Posterior dislocation of both shoulders is relatively rare and epileptic seizures has been considered as a common cause. Electrical injury is a rare cause of posterior shoulder dislocation. These cases are of special interest because most of them are missed on initial examination. Here we present a unique case of bilateral posterior dislocation of the shoulder with heterogeneous injury pattern- with one shoulder exhibiting reverse Hillsach's lesion and the other isolated lesser tuberosity fracture. We will discuss mechanism of injury, investigative parameters and treatment protocols followed.

Presentation of case: This report presents a case of bilateral posterior shoulder fracture dislocation after electrical shock. We were able to find a few individual case reports describing this condition. The case was acute and had a heterogeneous injury pattern of Reverse hill sach and Isolated lesser tuberosity fracture. Our treatment in this case consisted of closed reduction under general anesthesia of one shoulder and surgical reduction of other shoulder by McLaughlin procedure. Post operatively orthoses was used to keep the shoulders in abduction and external rotation. A rehabilitation program was begun after 3 weeks of immobilization. After 6 months of injury the patient has returned to work. 24 months postoperatively, at final follow-up, he was painless and capable of performing all of his daily activities.

Discussion: The amount of bilateral shoulder dislocations after electrical injury with the following injury pattern is not reported but is known to be very rare. The aim of this case presentation is to report an example for this rare entity, highlight the difficulties in diagnosis and review the treatment options.

Conclusion: Physical examination and radiographic evaluation are important for quick and accurate diagnosis. Early detection and treatment of such a condition is extremely important.
\end{abstract}

Keywords: Bilateral posterior fracture shoulder dislocation, electrical shock, reverse hill-sachs lesion, isolated lesser tuberosity fracture of humerus, mclaughlin procedure

\section{Introduction}

Posterior dislocation of both shoulders is relatively rare and epileptic seizures has been considered as a common cause. Although epilepsy, extreme trauma, and electric injuries are the proposed mechanism of injury, less than $5 \%$ of posterior shoulder dislocations are caused by electrical shocks ${ }^{[1]}$. These cases are of special interest because most of them are missed on initial examination. Despite the presence of pain, swelling and typical limitation of motion, false initial diagnosis is mentioned in retrospective reviews in the literature, in a rate of $50-80 \%[2,3]$.

Reverse Hillsach's lesions are the frequently associated with posterior dislocations. Isolated lesser tuberosity fractures of the proximal humerus following a posterior dislocation has not been mentioned in the available literature. This injury is more akin to isolated greater tuberosity fractures associated with anterior gleno-humeral dislocation. Here we present a unique case of bilateral posterior dislocation of the shoulder with heterogeneous injury pattern- with one shoulder exhibiting reverse Hillsach's lesion and the other isolated lesser tuberosity fracture. We would also like to discuss mechanism of injury, investigative parameters and treatment protocols followed.

\section{Case report}

40 year old patient with posterior dislocation of both the shoulders was referred to our hospital from peripheral clinic following an electrocution (low grade?) injury. 
Imaging was done (fig 1) and patient was treated with analgesics in peripheral nursing centre. In view of persisting shoulder pain and inability to move both the shoulders CT scan was done, which confirmed the diagnosis of Posterior fracturedislocation of Right shoulder, Posterior dislocation of Left shoulder was missed. Patient was later referred to tertiary care hospital for further management where the following diagnosis was confirmed.

On orthopaedic examination, we found that external rotation of both the shoulders was restricted and there was no evidence of peripheral nerve injuries. There were no associated injuries in other extremities.

CT scan was re-evaluated with the help of the radiologist to note the following findings-

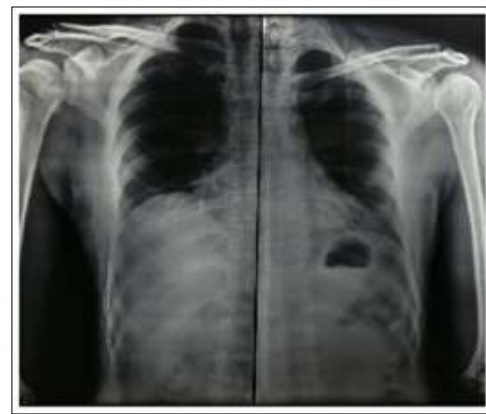

Fig 1.

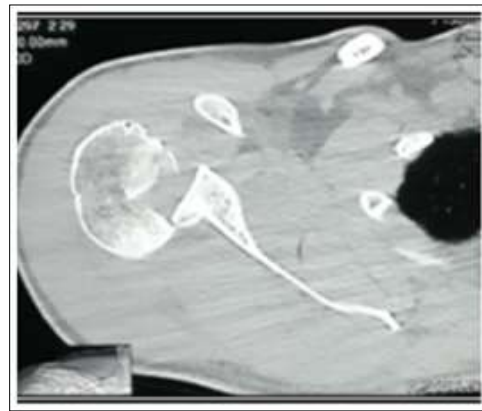

Fig 2.

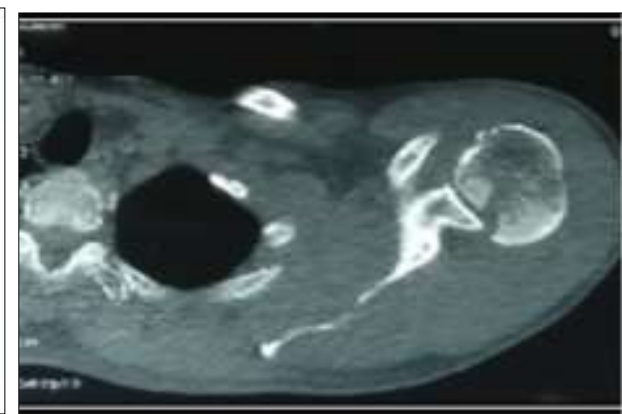

Fig 3.
Physicians evaluated the patient for ruling out the complications of electrocution. He was planned for reduction of shoulder under anaesthesia.

\section{CT scan}

Right shoulder CT was reported as a "displaced (probably inferiorly) two-part intra-articular fracture of the humeral head involving the lesser tuberosity" (Fig. 2)

As left shoulder injury was suspected, CT scan of left shoulder was done, reported as posterior fracture dislocation with McLaughlin lesion or Reverse Hill-Sachs lesion. (Fig. 3)

\section{Surgical procedure}

Patient was posted for surgery on the following day. Closed reduction was successful on the left shoulder with the reduction being stable as confirmed under C-Arm. Right sided shoulder reduction was attempted, however, reduction was unstable as noted under C-Arm. Right shoulder fracture dislocation was treated following McLaughlin's procedure. (Fig4) (Fig 5)

\section{Intraoperative pictures}

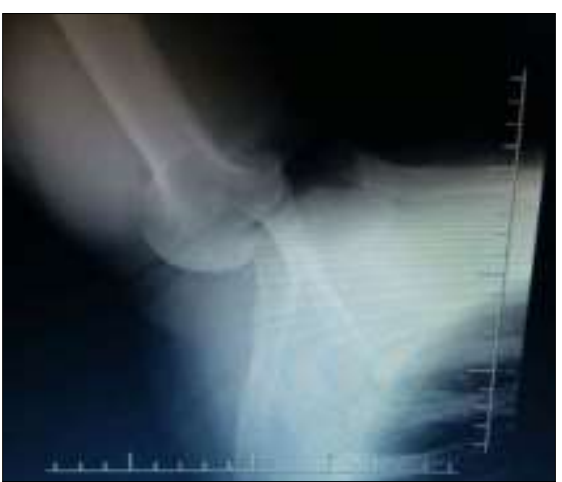

Fig 4: Right shoulder reduction achieved right

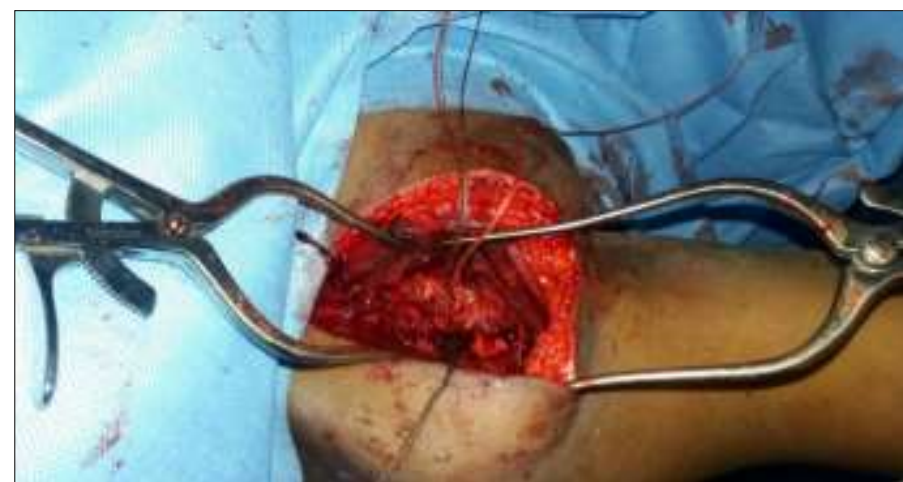

Fig 5: Open transfer of subscapularis tendon to fill the reverse Hill Sachs lesion (McLaughlin procedure)
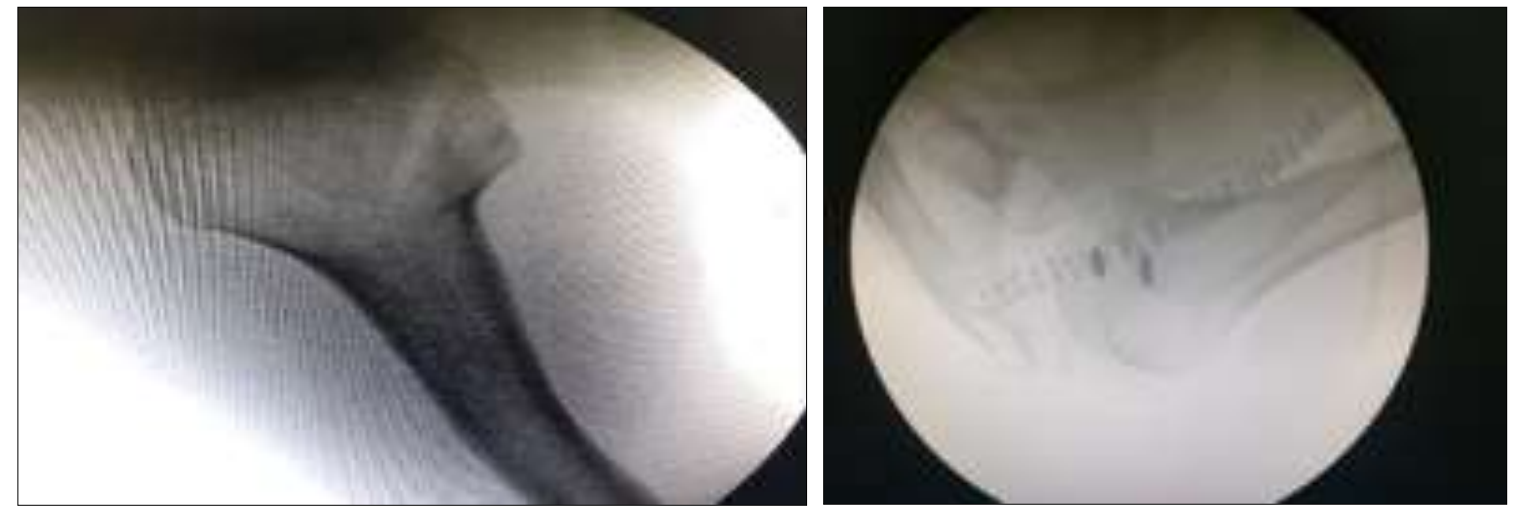

Fig 6: Post op x rays

\section{Post-operative protocol}

Post-operatively, bilateral shoulder were kept in 20 degrees of abduction with 10 degrees of external rotation using a gun slinger splint (Fig. 8) 


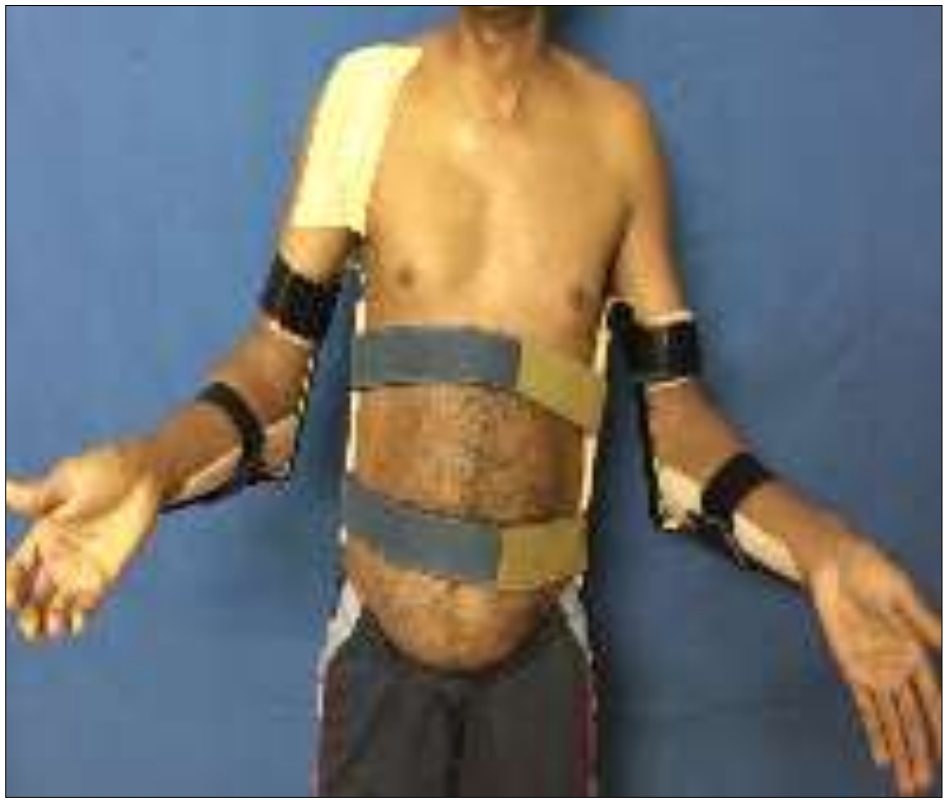

Fig 8.

\section{Follow up: Functional recovery}

On $5^{\text {th }}$ post-operative day, patient was discharged; staples were removed 2 weeks post operatively and physiotherapy in the form of gentle active and passive shoulder ROM exercises

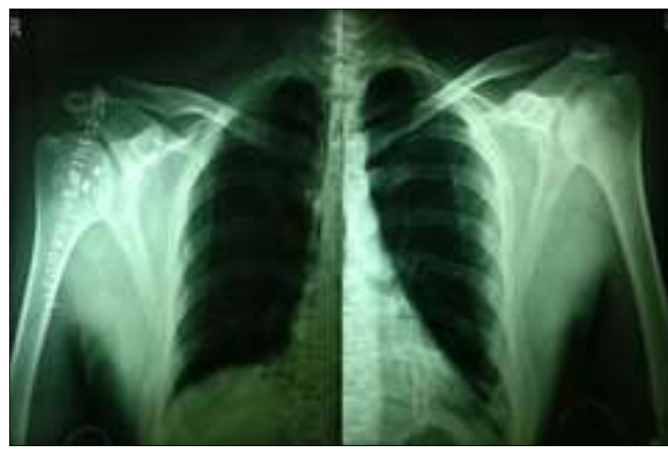

Fig 9: 2 Weeks post-operative

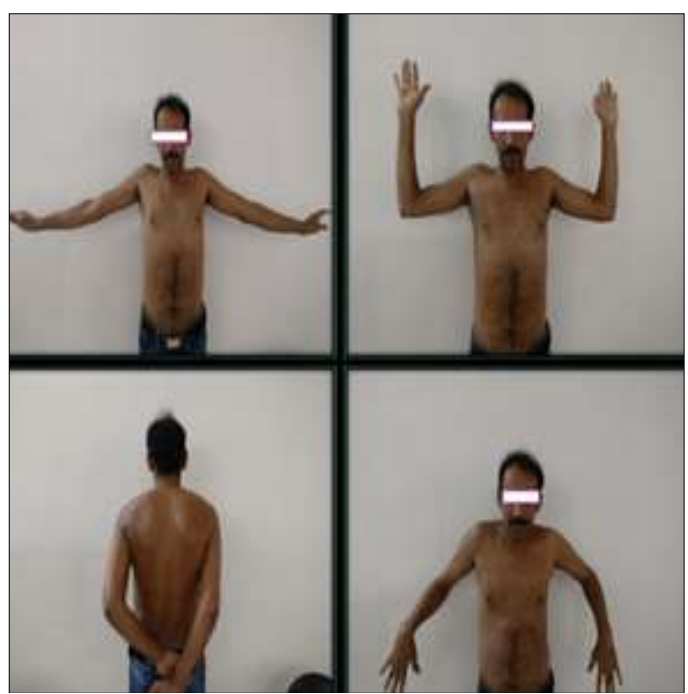

Fig 11: 6 Weeks post-operative ROM were initiated 4 weeks post operatively. The ROM of B/L shoulder joint at 6 weeks and 10 weeks post operatively are shown below. (Fig 9) (Fig 10)(Fig 11)(Fig 12)

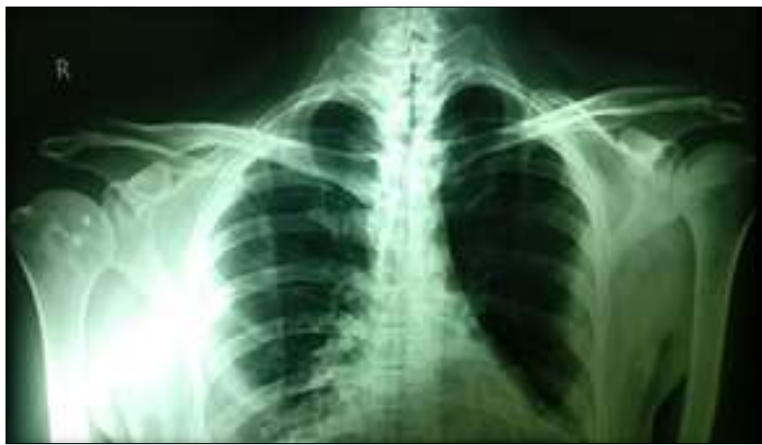

Fig 10: 6 Weeks post-operative

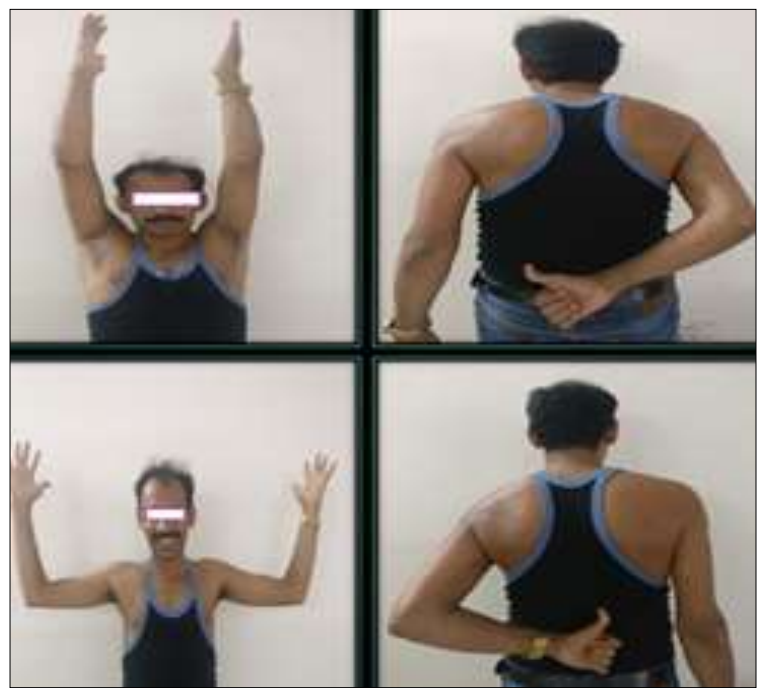

Fig 12: 10 Weeks post-operative ROM

Table 1: Post-operative rehabilitation, ROM: Range of motion; ORIF: Open reduction internal fixation

\begin{tabular}{|c|c|c|}
\hline Shoulder ROM progression & 10 weeks post-operative & 24 months post-operative \\
\hline \multirow{2}{*}{ Left side (ORIF) } & Flexion: $100^{\circ}$ & Flexion: $180^{\circ}$ \\
& Abduction: $90^{\circ}$ & Abduction: $160^{\circ}$ \\
\hline
\end{tabular}




\begin{tabular}{|c|c|c|}
\hline & External rotation: $35^{\circ}$ & $\begin{array}{c}\text { External rotation: } 80^{\circ} \\
\text { Internal rotation: } 45^{\circ}\end{array}$ \\
\hline & Internal rotation: $40^{\circ}$ & Flexion: $135^{\circ}$ \\
Abduction: $90^{\circ}$ \\
Right side (McLaughlin) & Flexion: $90^{\circ}$ & External rotation: $45^{\circ}$ \\
& Abduction: $90^{\circ}$ & Internal rotation: $50^{\circ}$ \\
\hline
\end{tabular}

\section{Discussion}

Bilateral posterior dislocation of the shoulder is in itself, a rare entity. Mynter first described it in $1902^{[1]}$. Neer later, identified fourteen cases of posterior shoulder fracture-dislocations out of 1500 dislocations overall $(0.9 \%)$. The most common cause resulting in such cases, is a convulsive seizure. Other causes mainly include trauma and electric shock ${ }^{(2)}$.McLaughlin also reported the mechanism of injury in posterior fracture dislocation. The rarity and high rate of misdiagnosis of this injury led McLaughlin to call it a "diagnostic trap" [4].

Posterior humeral dislocations often go undetected. Proposed explanations for the delay in diagnosis include failure of the evaluating physician to include the condition in the differential diagnosis, suboptimal radiographic evaluation and interpretation, and coincidental injuries such as fractures that can confound the patient's presentation. It is imperative that the orthopaedic surgeon develop a complete understanding of the nature of this injury and its treatment so that patients who present with this condition can be diagnosed and treated effectively. Hawkins et al. mentioned that only $30 \%$ of the cases of posterior dislocation are diagnosed within 6 weeks of injury; usually, $75 \%$ of the cases show a delay in diagnosis by an average of one year. A high index of suspicion, along with adequate imaging techniques are key in establishing a correct diagnosis ${ }^{[5]}$.

Hence, in our case the preferred modality used is a CT scan for diagnosing posterior dislocation as well as measuring the size of the articular defect/Reverse Hill Sachs.

In our case scenario we encounter two unique pathological shoulder presentations

\section{Reverse Hill Sachs lesion of right shoulder}

Mechanism of injury of Reverse Hill Sachs lesion aren't well described in many published literature. It is usually seen following shock or convulsion with shoulder in adduction, internal rotation and flexion. Below is a diagram describing the likely mechanism of injury of Reverse Hill Sachs lesion. (Fig 13)

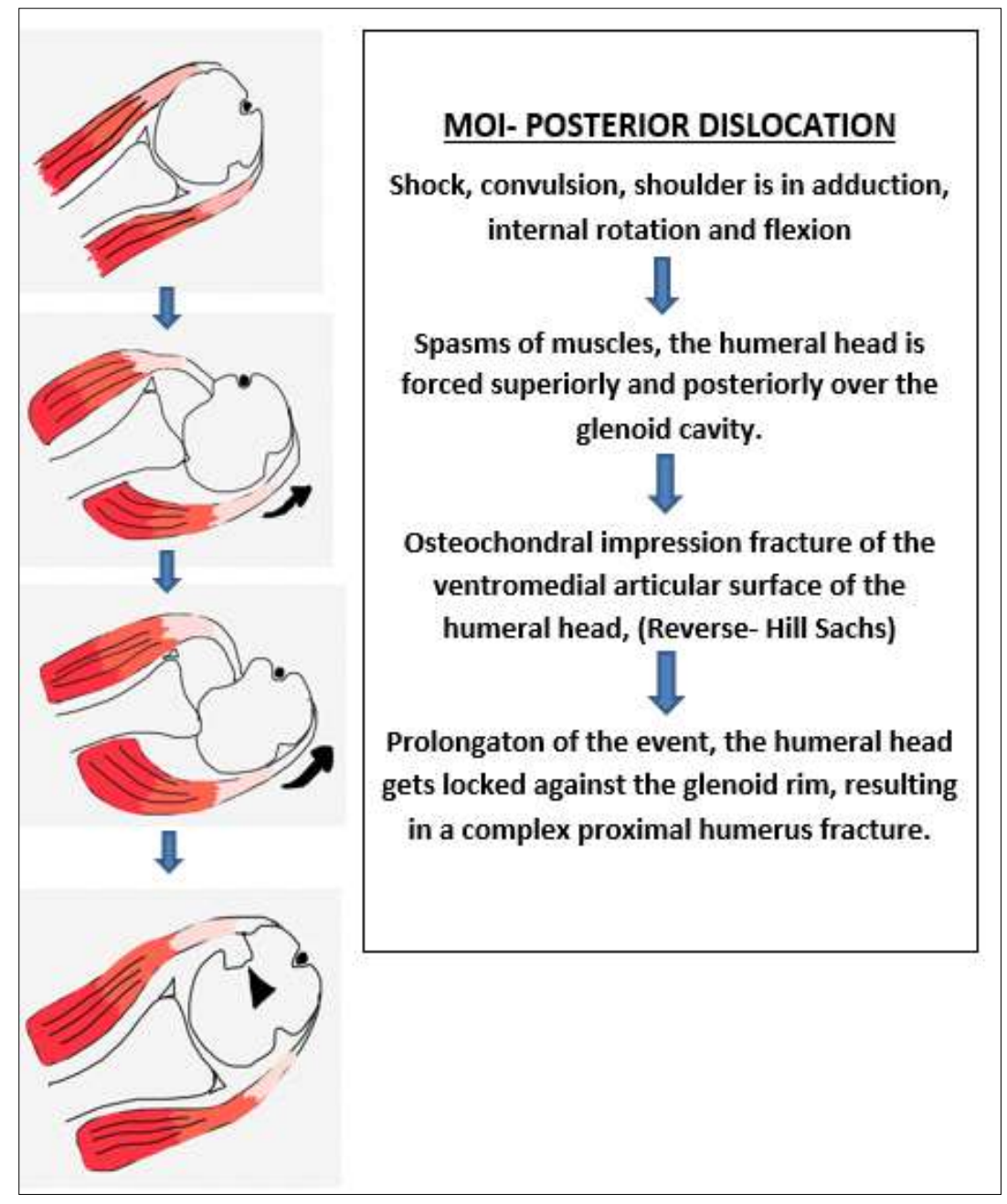

Fig 13: Schematic diagram of superior view of right shoulder demonstrates mechanism of injury in reverse Hill Sachs lesion

A Reverse Hill Sachs lesion serves as a propagation point, rendering the proximal humerus susceptible to fracture of the anatomic neck, either at the time of injury or with subsequent iatrogenic displacement during a reduction maneuver.
Secondly, the deformity of the humeral head articular surface resulting from this impression defect makes the glenohumeral joint susceptible to secondary osteoarthritis. Third, the size of the impression defect determines the stable arc of curvature of 
the glenohumeral articulation. As the defect enlarges, it is exposed to the posterior glenoid rim at an earlier point in the glenohumeral arc of motion, rendering the glenohumeral joint susceptible to redislocation. Hence early detection and treatment is important for such cases.

- Isolated Lesser Tuberosity fracture of Humerus of Left shoulder

Isolated lesser tuberosity of the humerus is a rare fracture which has not previously been described in the radiological literature. Mechanism of injury of such fractures involves application of a strong force to the arm when it is in a position of external rotation and abduction. The patient's arm was forcibly abducted and externally rotated with sudden contraction of the subscapularis and avulsion of the lesser tuberosity.

The subscapularis muscle arises from the anterior aspect of the body of the scapula and inserts into the lesser tuberosity (Fig. 14). With the arm in a position of $90 \sim$ abduction, the muscle is "relaxed". Further forced abduction and external rotation of the humerus, together with contraction of the muscle in the opposite direction (downwards and medially) combine to avulse the bony insertion in the lesser tuberosity. (Fig. 15)

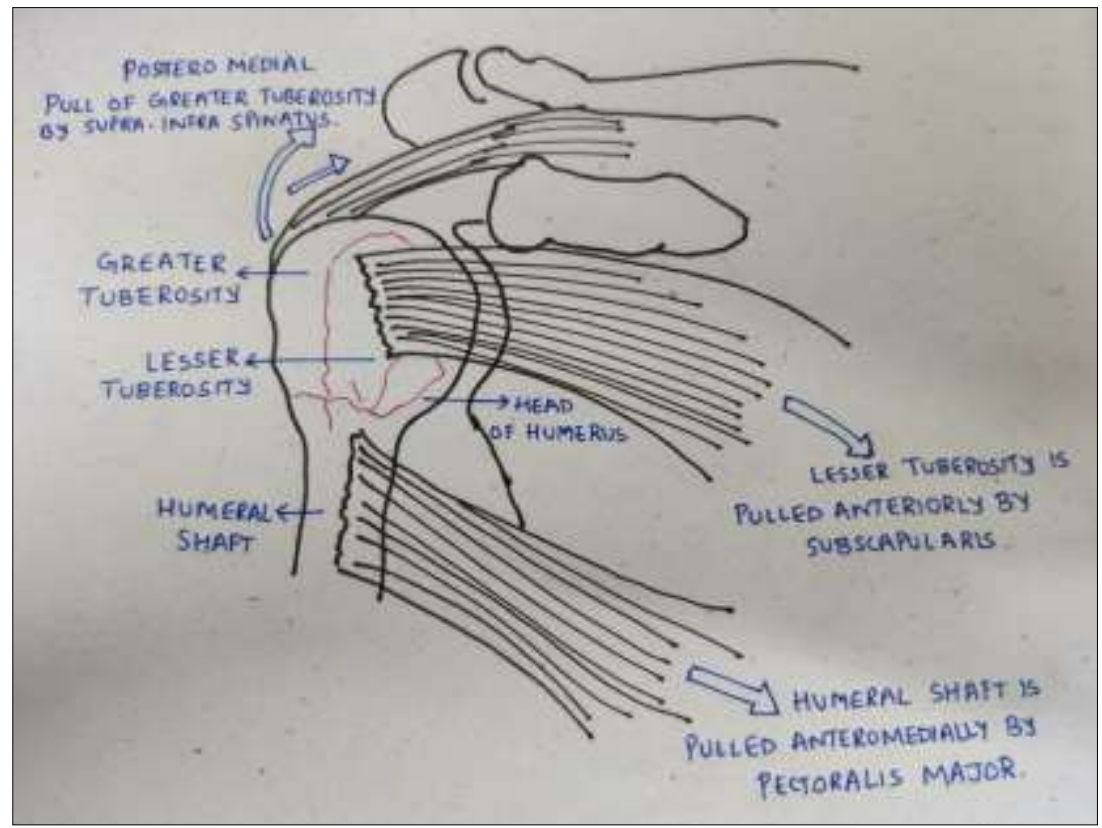

Fig 14: Attachment of rotator cuff muscles and forces acting on it.

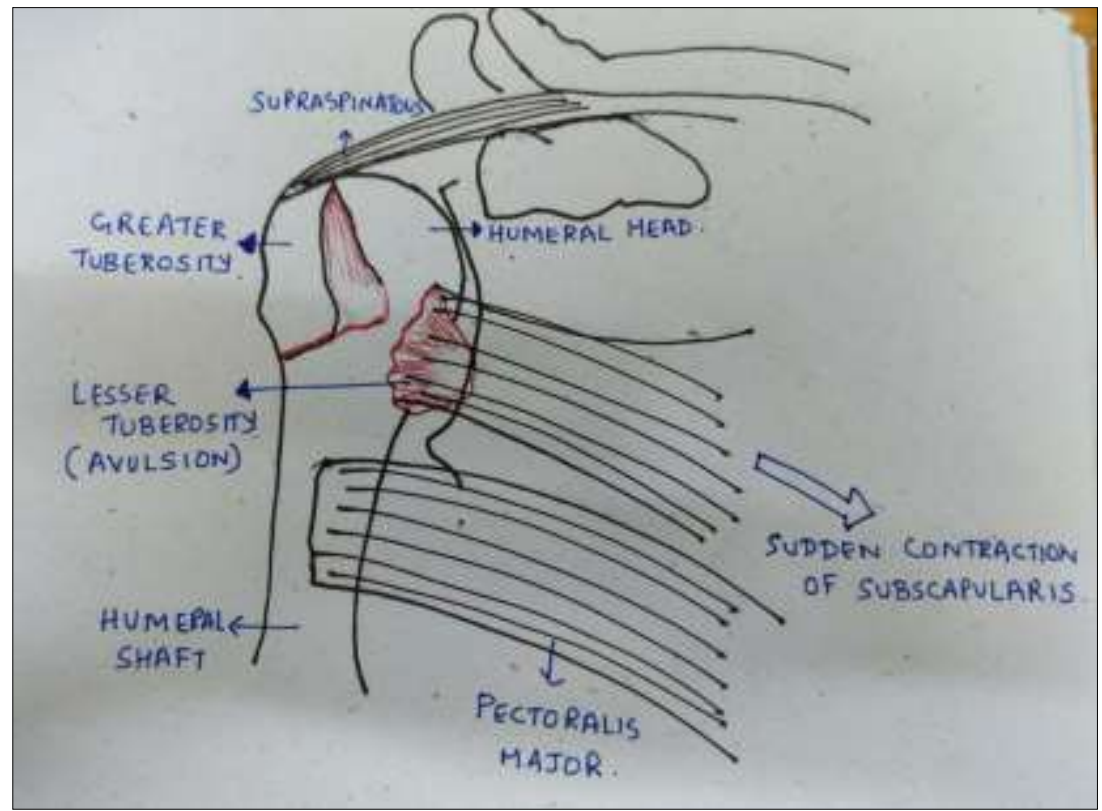

Fig 15: Mechanism of Injury showing isolated fracture of lesser tuberosity. 


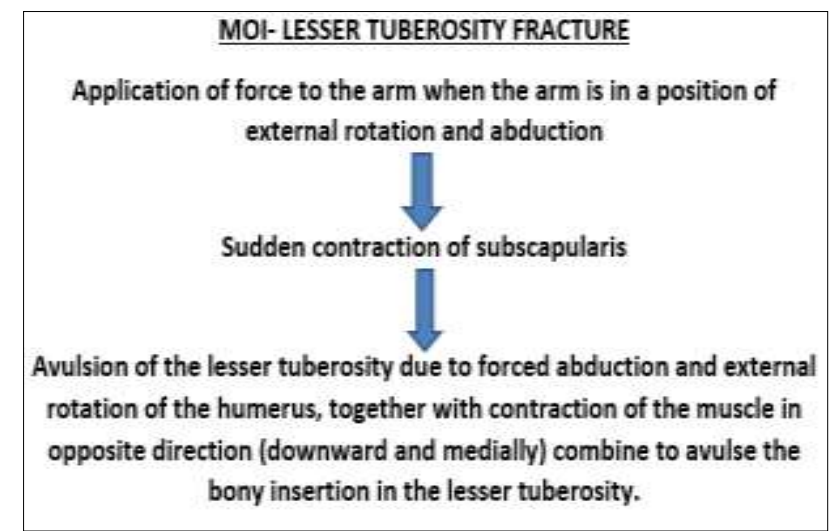

Delay in diagnosis and treatment has an adverse effect on the prognosis. Hence several treatment options have also been proposed to address this type of injury, ranging from nonsurgical methods to humeral head reconstruction procedures or arthroplasty with no clear consensus over definitive treatment guidelines, reflecting the complexity of this injury in addition to the limited evidence provided by the literature. However, there seems to be a consensus on the key factors determining the optimum treatment plan.

Key factors taken into consideration in the management of fracture-dislocation of the shoulder are the patient age, fracture type, extent of damage to articular surface, whether unilateral or bilateral, duration of dislocation and level of patient activity (HINDAWI).

Based on Kokkalis et al., a treatment algorithm for posterior shoulder fracture-dislocation was proposed (Fig 16) ${ }^{[13]}$.

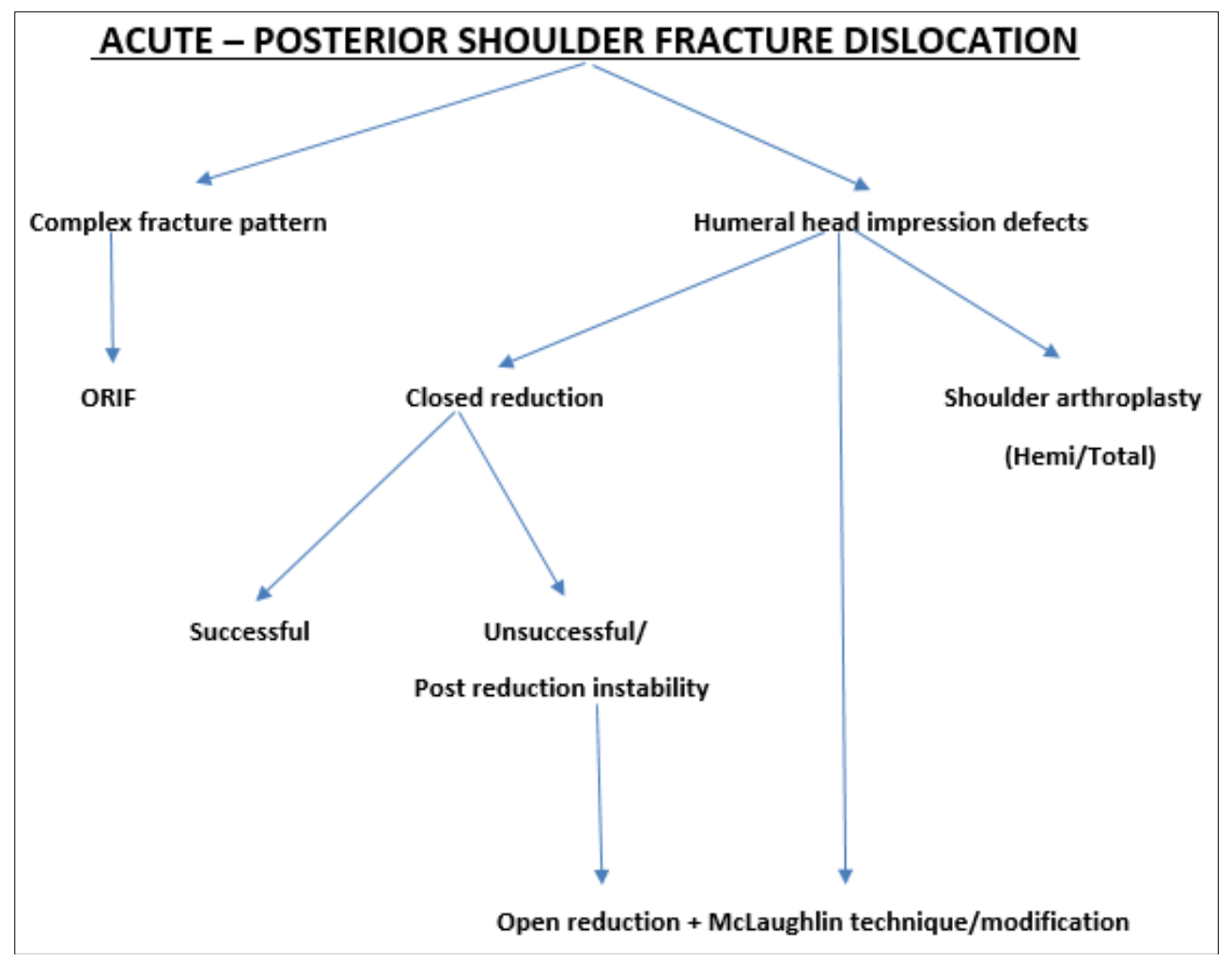

Fig 16: Treatment algorithm for posterior shoulder fracture -dislocation.

Closed reduction under general anesthesia has been shown to produce good results when performed in acute cases, treated in less than 3 weeks after the injury, and combined with a humeral defect of less than $25 \%$. In our case, Left shoulder fracture dislocation fulfilled the following criteria and hence closed reduction was attempted. Duralde et al. in their 2006 study reported excellent results for more than $70 \%$ patients with defects up to $27 \%$ treated acutely at about 2 weeks post-injury [14].

It is advocated that if the dislocation was less than 6 weeks old and the defect less than 20 per cent, a closed reduction can be attempted. If unsuccessful, a modified McLaughlin procedure should be performed ${ }^{[9]}$. If the injury is between 6 weeks and 6 months old, a modified McLaughlin can also be carried out. If the defect size is greater than 45 per cent or if the injury is older than 6 months, a hemi-arthroplasty or total shoulder replacement is recommended ${ }^{[11]}$. The condition of the glenoid is the deciding factor.

In our case, the patient presented with Posterior acute fracture dislocation of Right shoulder with Reverse hill sachs lesion involving up to $25 \%$ of the articular surface. Based on the algorithm, closed reduction was attempted. Due to unsuccessful reduction, McLaughlin procedure was done.

\section{Conclusion}

We present a rare case of bilateral posterior shoulder dislocation after electrical shock. Physical examination and radiographic evaluation are important for quick and accurate diagnosis. Bilateral fracture dislocation having a combination of Reverse Hill Sachs lesion on one shoulder and isolated lesser tuberosity fracture of the other shoulder are extremely rare and needs to be diagnosed accurately.

If the diagnosis of Reverse Hill Sachs is made early and the humeral head impression defect is up to $25 \%$, surgical reduction augmented by a McLaughlin procedure followed by a good rehabilitation program can lead to successful results.

\section{References}

1. Brackstone M, Patterson SD, Kertesz A. Triple "E" syndrome: Bilateral locked posterior fracture dislocation of the shoulders. Neurology 2001;56:1403-4.

2. Neer CS. II Displaced proximal humeral fractures. I. 
Classification and evaluation. J Bone Joint Surg. Am 1970;52(6):1077-1089.

3. Neer CS. II Displaced proximal humeral fractures. II. Treatment of three-part and four-part displacement. J. Bone Joint Surg. Am 1970;52(6):1090-1103.

4. McLaughlin HL. Posterior dislocation of the shoulder. J Bone Joint Surg. Am 1952;24:584-590.

5. Shaw JL. Bilateral posterior fracture-dislocation of the shoulder and other trauma caused by convulsive seizures. J Bone Joint Surg 1971;53A:1437-1440.

6. Karpinski MR, Porter KM. Bilateral posterior dislocation of the shoulder. Injury 1984;15:274-6.

7. Wadlington VR, Hendrix RW, Rogers LF. Computed tomography of posterior fracture-dislocations of the shoulder: Case reports. J Trauma 1992;32:113-5.

8. Kokkalis ZT, Iliopoulos ID, Antoniou G, Antoniadou T, Mavrogenis AF, Panagiotopoulos E. Posterior shoulder fracture-dislocation: an update with treatment algorithm. Eur. J Orthop. Surg. Traumatol 2016, P1-10.

9. Paul J, Buchmann S, Beitzel K, Solovyova O, Imhoff A. Posterior shoulder dislocation: systematic review and treatment algorithm. Arthroscopy.

10. Shaw JL. Bilateral posterior fracture-dislocation of the shoulder and other trauma caused by convulsive seizures. J Bone Jt Surg Am 1971;53:1437-1440.

11. Bhaga R, Aden AA. Results of the McLaughlin procedure for chronic locked posterior dislocation of the shoulder. SA Orthopaedic Journal 2009;8(1):53-9.

12. Din KM, Meggitt BF. Bilateral four-part fractures with posterior dislocation of the shoulder: a case report. J Bone Joint Surg. (Br.) 1983;65:176-178.

13. Kokkalis ZT, Iliopoulos ID, Antoniou G, Antoniadou T, Mavrogenis AF, Panagiotopoulos E. Posterior shoulder fracture-dislocation: an update with treatment algorithm. European Journal of Orthopaedic Surgery \& Traumatology 2017;27(3):285-94.

14. Duralde XA, Fogle EF. The success of closed reduction in acute locked posterior fracture-dislocations of the shoulder. Journal of shoulder and elbow surgery 2006;15(6):701-6. 